\title{
Integral Control and Dead Zoom Phenomenon Analysis for System with Unknown Bounded Function
}

\author{
Wenguang Zhang ${ }^{1, a}$, Lingling Wang ${ }^{1}$ and Tian Lan $^{2}$ \\ ${ }^{1}$ Department of control engineering, Naval aeronautical and Astronautical university Yantai of China, \\ 264001 \\ ${ }^{2}$ Department of strategy and campaign, Naval command college, Nanjing of China, 264001 \\ ${ }^{a}$ Zwg1024@126.com
}

Keywords: Bounded function, dead zoom, integral control, PID control, stability

\begin{abstract}
A kind of uncertain nonlinear system with unknown bounded function is analyzed. And a kind of common integral control is used to cope with the unknown function by taking example of a first order system. And by using of Laypunov stability theorem, we find that the system is stable but the unknown parameter is difficult to estimate and dead zoom will be exist no matter how big control gain is chosen. At last, detailed simulation result is done and the main result of this paper is testified.
\end{abstract}

\section{Introduction}

The research of uncertain system control not only causes the attention of many scholars in theory[1-4], but also is inevitable in engineering. Because the actual control system is more or less with some uncertainty, and therefore the nature of the control is to eliminate the bad effects of the system caused by uncertainties and unknown disturbance of the system performance[5-7]. There are many different forms of the mathematical description of the system, and the uncertain nonlinear function is the most common one. This paper is aimed at this kind of bounded unknown nonlinear function, and carries on the design of integral adaptive control. Through the analysis, it is indicated that the stability of the nonlinear system is bounded, and the dead zone is inevitable. Although the conclusion is obtained by the simple first order system simulation, it is significant to the high order nonlinear system, because it reveals the nature of the control of nonlinear system.

\section{Model Description}

Consider the bellow first system

$$
\dot{x}=f(x)+u
$$

Where $f(x)=a_{1} x+a_{2} \sin x+a_{3} x^{2}$, and $f(x)$ not only contains unknown parameters and also the mode function is also unknown.

And the control object is to design a PID control law such that the state can converged to expected value $x^{d}$, where $\dot{x}^{d}=0$.

\section{Analysis of Integral Control Effect for System with Unknown Mode Function}

Define a error variable as

$$
z=x-x^{d}
$$

Then

$$
\dot{z}=\dot{x}-\dot{x}^{d}=\dot{x}=f(x)+u
$$

Then design a PI control law as

$$
u=-k_{p} z-\hat{D}_{0}
$$

If we assume $|x| \leq D$, and since unknown mode function is bounded, then it holds 


$$
|f(x)| \leq D_{0}
$$

Then it can be written as

$$
\dot{z}=f(x)-k_{p} z-\hat{D}_{0}
$$

With proper transformation, it holds:

$$
z \dot{z}=z f(x)-k_{p} z z-z \hat{D}_{0}
$$

And with proper reduction method, it satisfies

$$
z \dot{Z}=z f(x)-k_{p} z z-k_{i} z \int z d t \leq|z| D_{0}-k_{p} z z-z \hat{D}_{0}
$$

Define $\tilde{D}_{0}=\operatorname{sign}(z) D_{0}-\hat{D}_{0}$, then if $z \neq 0$, it has

$$
\dot{\tilde{D}}_{0}=-\dot{\hat{D}}_{0}
$$

Then

$$
z \dot{Z} \leq z\left(\operatorname{sign}(z) D_{0}-\hat{D}_{0}\right)-k_{p} z z=-k_{p} z z+z \tilde{D}_{0}
$$

And design the turning law of unknown parameters as

$$
\dot{\hat{D}}_{0}=k_{d} z
$$

Then choose a Lyapunov function as

$$
V=\frac{1}{2} z^{2}+\frac{1}{2 k_{d}} \tilde{D}_{0}^{2}
$$

then

$$
\dot{V}=z \dot{Z}+\frac{1}{k_{d}} \tilde{D}_{0} \dot{\tilde{D}}_{0}=z \dot{Z}-z \tilde{D}_{0} \leq-k_{p} z z \leq 0
$$

So the system is stable.

So if we do not use adaptive method, or even we use adaptive method but the unknown parameter can not be estimated accurately, then there must exists a dead zone since most nonlinear systems are not global stable. So it means that most nonlinear systems are stable in finite range and there exists a dead zone.

\section{Simulation Analysis}

We assume the initial value of state is 0 , and $a_{1}=1, a_{2}=2, a_{3}=3$, and the expected value is set as $x^{d}=2$, and a matlabe program can be written as following with simulink toolbox:

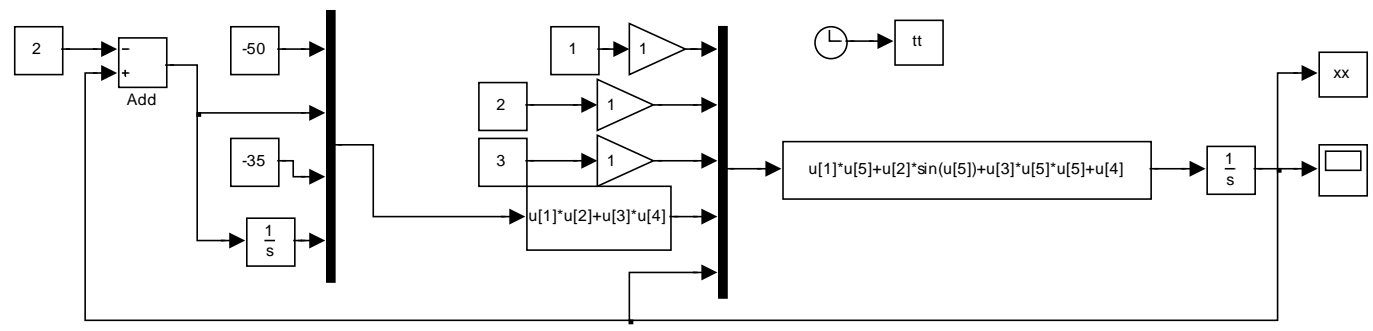

Fig. 1. Program structure with simulink And simulation result can be shown as following figures 


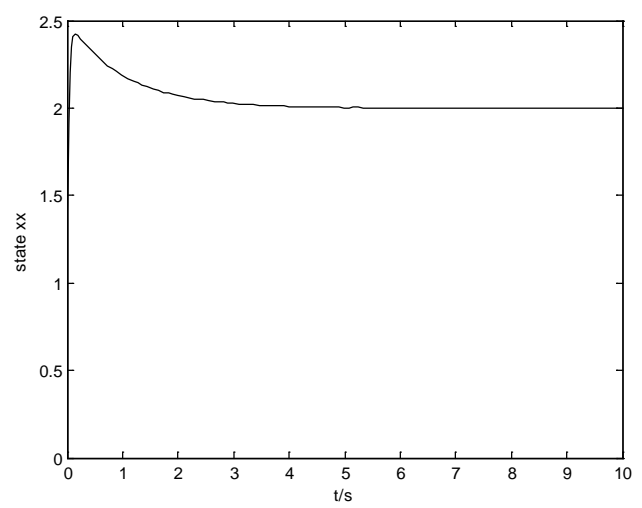

Fig.2. The curve of system state

And if we increase the expected value as $x^{d}=4.7$, then the system is unstable, the simulation result is shown as bellows:

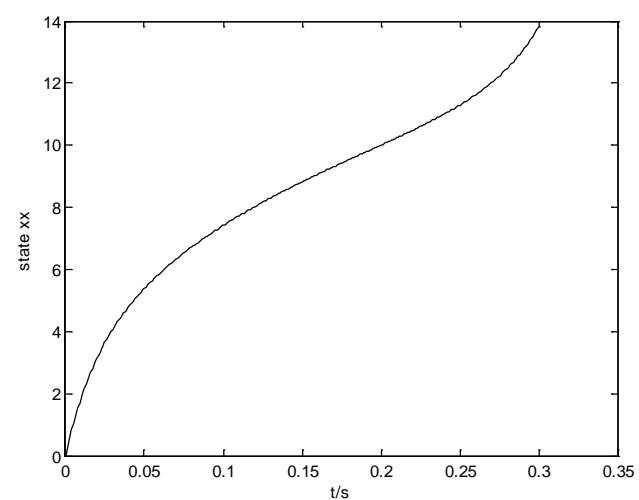

Fig.3. The unstable curve of system state

So it is shown that the system is stable in finite interval. And we set a small input signal such as $x^{d}=0.0001$, and simulation result is shown as below Fig.4.

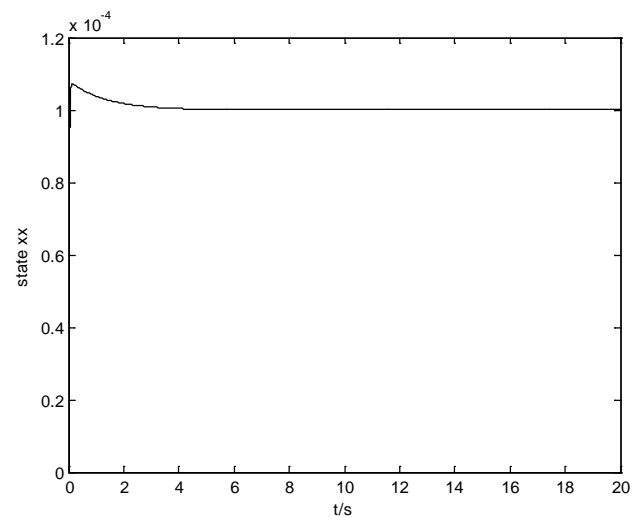

Fig.4. The stable curve of system state

So the dead zone phenomenon is not very clear. Then we set $f(x)=a_{1} x+x^{1 / 3}+a_{2} \sin x+a_{3} x^{2}$, then simulation result can be shown as 


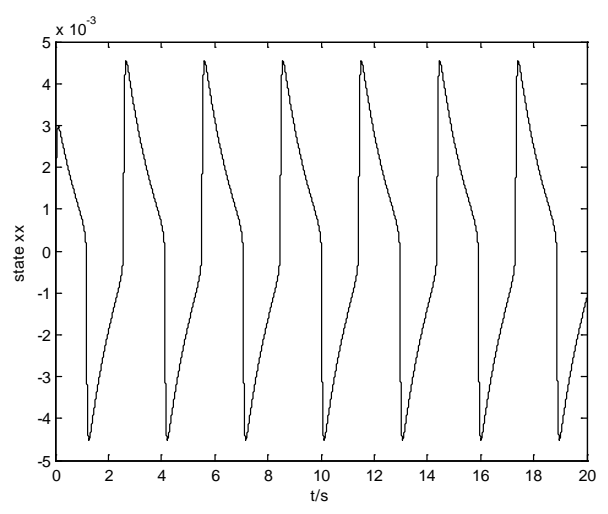

Fig.5 Dead zone phenomenon

\section{Conclusion}

Through a simple first-order uncertain nonlinear system analysis, a kind of integral adaptive stabilizing controller is designed, and stability analysis achieved the following conclusions: the stability of the nonlinear system is bounded by the Lyapunov function method, and uncertainty bounds is difficult to achieve accurate estimation, especially that the dead zone is always exists. Finally, the correctness of the conclusion is verified by detailed simulation analysis. And because the conclusion reflects the nature of the nonlinear system, it is still significant to the high order nonlinear system.

\section{References}

[1] J.S. Uhlman, N.E. Fine, D.C. Kring. Calculation of the Added Mass and Damping Forces on Supercavitating Bodies. The 4th International Symposium on Cavitation,California,2001:7 13

[2] D.Clarke. Calculation of the Added Mass of Elliptical Cylinders in Shallow Water. Ocean Engineering.2001,28(4):61 72

[3] C.J. Atkinson, R.G. Urso. Modeling of Apparent Mass Effects for the Real-Time Simulation of a Hybird Airship. AIAA Modeling and Simulation Technologies Conference and Exhibit,Keystone.2006:21 32

[4] Yokomaku Y. The Stratospheric Platform Airship R\&D Program of Japan. The 2nd Stratospheric Platform Systems Workshop, Tokyo Japan.2000:7 13

[5] S.P. Jones, J.D. Laurier. Aerodynamic Estimation Techniques for Aerostats and Airships. AIAA Lighter-than-Air Systems Conference,Annapolis,2004: 88 94

[6] M.T. Soylemeza, N. Munrob, H. Bakic. Fast Calculation of Stabilizing PID Controllers.Automatic,2003,39(7):121 126

[7] Etkin B, Theory of the flight of Airplanes in Isotropic Turbulence Review an Extension, AGARD Rept.1961:372 